\title{
Maximising spectral efficiency in LTE cells
}

\author{
Tony Keenan, Rudi Villing
}

Callan Institute, Department of Electronic Engineering, National University of Ireland Maynooth.

email : tkeenan@eeng.nuim.ie,rudi.villing@eeng.nuim.ie

\begin{abstract}
The efficiency with which spectrum is used in wireless communications systems is becoming increasingly important as a result of the expected growth in traffic demand and the finite nature of usable spectrum. Spectral efficiency, defined as throughput divided by bandwidth, is a useful metric for evaluating the use of spectrum in wireless systems. In any given area the achievable spectrum efficiency is impacted by the underlying user population. This paper presents the methodology for finding the transmit power which maximises the spectral efficiency of a LTE cell for a given user density and traffic type. The impact of different user densities and traffic profiles on the choice of transmit power is evaluated. Results show that the transmit power which maximise spectral efficiency decreases as the user density and average data rate of the traffic profile increases. Two non ideal real world scenarios which require an increase in cell spectral efficiency are also considered and a modified user admission scheme which can increase the cell spectral efficiency is presented and evaluated. Results showed that the spectral efficiency was improved but the maximum improvement depended on the traffic profile and practical constraints of the LTE standard.
\end{abstract}

Keywords - LTE, Spectral optimization, User population, Cell capacity, User coverage

\section{INTRODUCTION}

As wireless communication systems have evolved from low data rate services such as voice and SMS to high data rate services such as IP traffic and video streaming mobile traffic has increased significantly. According to [1] global mobile data traffic is expected to increase from 0.6 to 10.8 Exabyte's per month by 2016, an 18 fold increase. The traffic increase is a consequence of the growth in both the traffic per device and the number of wireless devices. It is estimated that by 2017 there will be 7 trillion wireless devices worldwide serving 7 billion users [2]. The usable spectrum available to mobile operators, however, is a finite resource and for this reason there is a need to utilize the available spectrum as efficiently as possible.

A common metric for evaluating the efficiency with which spectrum is used by a cell is the spectral efficiency, calculated as the total cell throughput divided by the bandwidth used. Modern wireless standards such as Long Term Evolution (LTE) [3] improve spectral efficiency (over legacy standards) through the use of advanced modulation and coding techniques and spatial multiplexing techniques using multiple input multiple output (MIMO) communication.

Due to the constrained nature of spectrum resource there is a growing body of literature focusing on the evaluation and improvement of spectral efficiency in modern wireless standards. For example, the analytically derived results of [4] indicated that the spectral efficiency of Orthogonal Frequency Division Multiple Access (OFDMA) cells was constant and independent of distance when inter-cell interference was not considered. The analysis did, however, ignore the effect of population distribution and traffic mix. Relay enhanced cells for LTEAdvanced were investigated in [5]. Analytically derived results indicated that spectral efficiency improved by $14 \%$ when cells used three relay nodes in addition the base station. The improvement arose because relay nodes permit users further from the base station to use higher order modulation schemes than would otherwise be possible. Again the analysis did not examine the effect of population or traffic.

Although it is possible to calculate theoretical spectral efficiency independently of any population or traffic considerations, this calculated value may not represent real world values very well. When population and traffic are not directly considered, there is an implicit assumption that the cell will utilize its full spectrum allocation regardless of cell radius. Therefore, if there are fewer users (due to a small cell radius for example), then each user must be assumed to generate more traffic. In practice it may be more realistic to analyse the spectral efficiency of a cell for a specific traffic profile (or mix of profiles) and such analysis requires consideration of the population distribution also. For example, [6] found that the cell capacity for mobile WiMax varied significantly with the users traffic profile and user admission scheme. It was found that the number of users could be increased by a factor of nine through the use of smart resource scheduling. An increase in the number of users directly impacts the spectral efficiency that can be 
achieved for a given traffic profile. Nevertheless, their analysis still did not consider population distribution.

The remainder of this paper presents the methodology for determining the transmit power which maximises the spectral efficiency of an LTE cell given a population of active users. Unlike the literature cited above, this work considers the effects of both traffic profile and population distribution. User admission depends on traffic profile and may result in some time/spectrum resources being unused or underutilized for a variety of reasons [7] with a consequent negative effect on spectral efficiency. For a given traffic profile (or traffic mix) the total traffic demands in the cell will increase or decrease with cell population which depends on cell area. It should be clear that for sufficiently small areas, the total traffic demand will not consume all of the cell's time/spectrum resources. Conversely, for sufficiently large areas, the total traffic demand cannot be satisfied.

The following section examines the transmit power (and cell area) which optimizes spectral efficiency given a population and traffic profile. Thereafter techniques for optimizing the spectral efficiency of non-ideal real world scenarios which require a modified population or cell area are considered.

\section{OPTIMIZING SPECTRAL EFFICIENCY}

In this section the model developed in [7] is used to evaluate spectral efficiency for different user densities and traffic profiles. The transmit power which maximises spectral efficiency for a given population and traffic profile is found and the impact of user density and traffic profile on the choice of transmit power is examined.

\section{a) Model Description}

The model developed in [7] allows simulation of a single LTE cell with a specific transmit power, population distribution, and traffic profile. The population is assumed to be uniformly distributed and is therefore characterized by its density. A traffic profile is characterized by the packet size, packet interval, and maximum acceptable latency. A simulation run using the model proceeds as follows. First the serviceable area is determined for the specified transmit power (based on the propagation model and the minimum SNR at which LTE communication can take place). All users within the serviceable area are assumed to want service and this set of users is referred to as the cell population. Next, users are selected at random from the cell population and admitted if their traffic demands can be met (subject to the maximum acceptable latency). This continues until either all users in the cell population have been admitted or there are insufficient control or data resources available to satisfy the user's traffic demand. At this point the simulation ends and a number of metrics are measured or calculated.

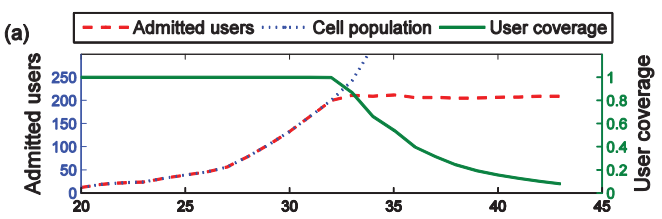

(b)

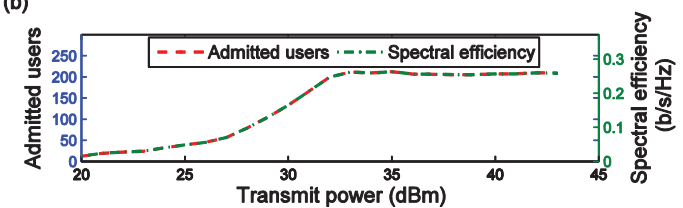

Figure 1: Relationship between transmit power and (a) number of users admitted, cell population, and user coverage, and (b) spectral efficiency

The total number of users that have been admitted is measured and the user coverage, the ratio of admitted users to the cell population, is calculated. Thereafter, the spectral efficiency, $S_{c}$, is calculated using

$$
S_{c}=\frac{N_{u} \times P}{T_{P} \times B}
$$

where $N_{u}$ is the number of users admitted, $P$ is the fixed packet size, $T_{P}$ is the fixed interval between packets, and $B$ is the bandwidth allocated to the cell. This is simply the total throughput of the cell divided by bandwidth used. Furthermore, it should be clear that the spectral efficiency depends on both the number of admitted users (which depends on cell population and serviceable area) and the traffic profile (via $P$ and $T_{P}$ ).

As in [7] only downlink communications without spatial multiplexing were considered and similar general simulation parameters were used. This included a noiseless channel with a bandwidth of 10 MHz. In addition a time division duplex (TDD) LTE frame with uplink-downlink configuration 1 in [8] was considered. The control section of each LTE subframe consists of four OFDMA symbols with each user requiring four control channel elements per data burst. Like [7] the free space path loss model was used [9]. Furthermore, a macro base station model was used in all simulations, regardless of transmit power.

\section{b) Optimum spectrally efficient transmit power}

Figure 1a shows the relationship between transmit power, cell population, number of admitted users and user coverage for a rural user density (2 users $/ \mathrm{km}^{2}$ ) and a VoIP traffic profile ( 31 bytes every $20 \mathrm{~ms}$ with $60 \mathrm{~ms}$ maximum latency). Once the traffic demands of the cell population exceeds the capacity of the cell the user coverage decreases as is clearly shown in the figure. The maximum number of users that can be admitted changes depending on the user traffic profile or mix of traffic profiles, the user admission scheme used, and the spatial distribution of users in the cell. Once this maximum is reached the number of users admitted remains constant as transmit power is increased. However both the total cell 
population and serviceable area of the cell continue to increase and as a result the user coverage decreases.

Figure $1 \mathrm{~b}$ shows the change in spectral efficiency with transmit power. As transmit power increases, spectral efficiency also increases in proportion to the number of users admitted as characterized by equation (1). As with the number of admitted users, once the cell capacity has been reached, increasing the transmit power further has no effect on spectral efficiency.

Subject to the constraint that the traffic demands of the full cell population must be met (i.e. user coverage must be 1), the optimum spectrally efficient transmit power, $P_{\text {Opt-SE }}$, is the one which maximises spectral efficiency. This is also the transmit power which maximizes the number of admitted users while still having a user coverage of 1 . At lower transmit powers, spectral efficiency is less than the maximum and the cell is using valuable spectrum resources inefficiently. At higher transmit powers, user coverage is less than 1 and the cell is unable to meet the traffic demands of the cell population. It is worth noting that $P_{O p t-S E}$ also provides the largest serviceable area that has full user coverage, thus requiring the smallest number of cells to cover a large geographical area.

For a fixed bandwidth, single base station scenario, it is important to note that the ability to provide full user coverage is sensitive to user density and traffic profile. For example, given a VoIP traffic profile, the maximum user density for which full coverage can be achieved in a macro cell model is approximately 30 users $/ \mathrm{km}^{2}$. At higher densities even the lowest possible transmit power results in a cell population which exceeds the capacity of the cell. Therefore, for higher densities an increase in either bandwidth or the number of base stations within a cell is required to provide full user coverage. (In fact, user coverage also depends on the base station and propagation models so that in significantly higher density suburban or urban environments full coverage may still be achieved.) Changes in the traffic mix associated with users have a similar effect to changes in user density.

\section{c) Effect of user density and traffic profile}

Using the methodology described above the optimum spectrally efficient transmit power can be calculated for different user densities and traffic profiles. Figure 2 a shows how $P_{O p t-S E}$ varies with user density assuming a constant VoIP traffic profile. The simulated line represents the optimum values determined from simulations. The ideal line represents values calculated analytically from knowledge of the user density and cell capacity using link budget analysis. Both lines match closely and the visible differences are largely due to the use of continuous power levels when calculating the ideal values in contrast to the discrete power levels used in simulations. The clear trend is that $P_{\text {Opt-SE }}$, and therefore cell size, decreases gradually as user density increases. This trend is consistent with the fact that (a)

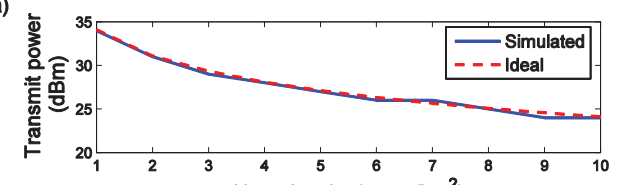

(b)

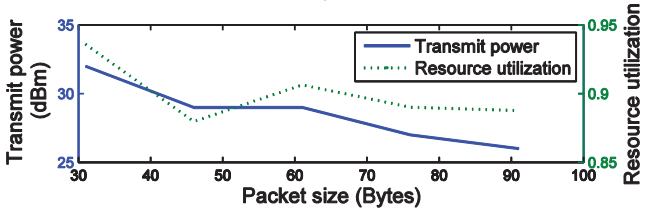

Figure 2: Optimum spectrally efficient transmit power for (a) fixed VoIP traffic profile with varying user density and (b) varying traffic profile with fixed

the total traffic demand within a given area increases as user density increases, due to a larger cell population. Because the cell populations are larger at any given transmit power, the range of transmit powers which permit full coverage reduces.

Figure $2 \mathrm{~b}$ presents the relationship between $\mathrm{a}$ varying traffic profile and $P_{O p t-S E}$ when the user density was held constant ( 2 users $/ \mathrm{km}^{2}$ ). The traffic profile was varied by taking the VoIP profile but increasing the packet size. Similar to increases in user density, increases in the traffic profile's packet size increase the traffic demand in a given area. As before, the result is that $P_{O p t-S E}$ decreases as traffic demand (in this case packet size) increases. The rate of decrease differs from that for user density, however.

The source of this difference is variations in the maximum level of resource utilization that can be achieved for different packet sizes. There are a number of reasons for such variations which result from unused or unallocated resources. These include the granularity with which LTE frame resources are allocated, the control overhead per packet, and the number of resources remaining when resources have been allocated to the maximum integer number of packets. For different packet sizes the number of unused resources changes. In particular, where the rate of decrease in transmit power with packet size (Figure 2a) slows relative to the corresponding decrease in user density (Figure 2b), the resource utilization increases (i.e. the number of unused resources decreases).

In summary, the trend in both Figure $2 \mathrm{a}$ and Figure $2 \mathrm{~b}$ is that the $P_{O p t-S E}$ decreases as the traffic demand increases, whether that increase is due to increased population or increased traffic demands from each user. This reduction in transmit power corresponds to smaller cell sizes and is consistent with the current philosophy of using smaller cells, for example femto cells, to provide higher capacity in a given area [10].

\section{OPTIMIZING FOR MODIFIED TRAFFIC OR AREA}

In the previous section $P_{O p t-S E}$ was determined for a range of user densities (based on a rural population) and traffic profiles (based on a VoIP profile). In a real 


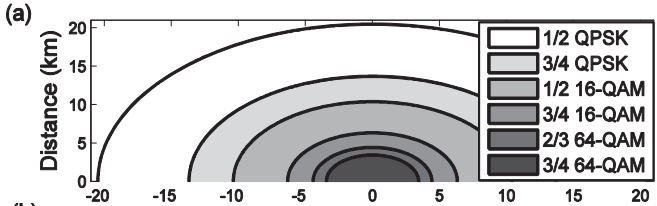

(b)

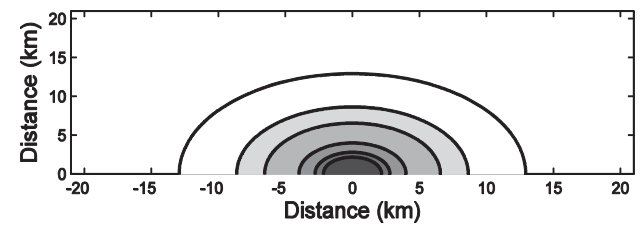

Figure 3: MCS range for (a) $43 \mathrm{dBm}$ and (b) 39 $\mathrm{dBm}$ transmit powers

world implementation it may not be practical to use the calculated optimum values for a variety of reasons. In this section, two principal reasons why the optimum value cannot be used are considered: first, the user density may change after the cell has been deployed, and second, the cell may be required to service an area other than the spectrally efficient optimum.

In the previous section the transmit power was chosen to maximise spectral efficiency for a particular total traffic demand (resulting from the combination of user density and traffic profile). The optimum value changes as traffic demand increases or decreases, but it may not be practical to change the cell area (which is the normal consequence of changing transmit power) after the cell has been deployed. Nevertheless, real world populations are expected to experience gradual increases in traffic demand over time [1]. Typical approaches for dealing with large changes in the underlying population density include increasing the bandwidth allocated to a cell or using additional micro or femto cells which can be turned on during times of increased demand [10]. However these solutions involve the cost of additional infrastructure or require the availability of additional spectrum which is not always possible. An alternative approach which does not require additional infrastructure or spectrum would be desirable, particularly for handling small or temporary changes.

The work thus far also assumes that for a given total traffic demand, a base station with a cell size corresponding to $P_{\text {OptSE }}$ can deployed. However, for network planning reasons this optimum size cannot always be used. Large, coarse changes can be handled by changing the bandwidth allocation of the cell (if suitable spectrum is available) or adding infrastructure such as relay nodes [5]. Again, for this reason, it would be desirable to have a mechanism for maximizing the spectral efficiency (and minimizing the power consumption) at a range of cell sizes without requiring changes to spectrum allocation or infrastructure.

\section{a) A modified user admission scheme}

Each modulation and coding scheme (MCS) of LTE has a corresponding MCS range, the maximum

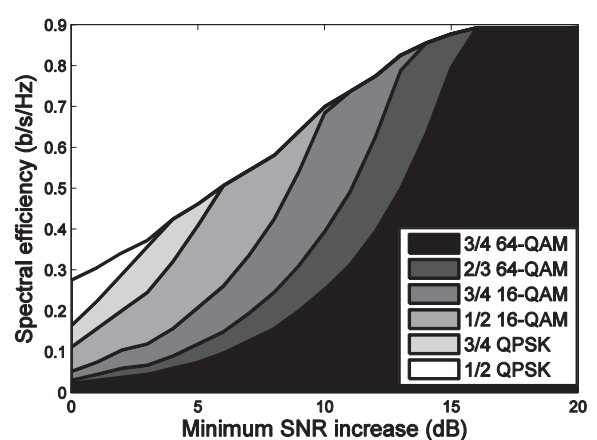

Figure 4: Spectral efficiency versus the increase in minimum SNR. Each area shows the relative contribution of that MCS to the total.

distance from the base station at which it can be used by a user. The MCS range can be calculated from the transmit power of the base station and the receiver signal to noise ratio (SNR) requirement of the MCS using a link budget analysis. (As a simplification, this work does not consider inter cell interference.) From the MCS ranges, the area which each MCS occupies can be calculated as a fraction of the total cell area. Figure 3 shows these distances for two different transmit powers. It can be shown that the fraction of the cell occupied by each MCS is independent of transmit power and cell radius. This is apparent in Figure 3 and is consistent with the results presented in [4] when inter cell interference is not considered.

The relative contributions of the various MCS areas to the total cell area determine the maximum spectral efficiency of the cell (assuming a uniform spatial distribution of users and random user admission). This leads to the results shown in Figure 1 where the number of users admitted and spectral efficiency reaches a maximum which is not exceeded as transmit power is increased.

The maximum spectral efficiency can be increased by increasing the minimum SNR threshold which users must reach in order to be admitted to the cell. In the default model, this threshold is equal to the minimum receiver SNR requirement of the lowest order MCS. By increasing this minimum SNR threshold, the fraction of users using the lowest order MCS will decrease. This results in more resources being available to higher order MCS users. Furthermore, resources allocated to a higher order MCS can carry more data than those allocated to a lower order MCS. As a consequence, either the existing higher order MCS users can generate more traffic, or more high order MCS users can be serviced. The result in either case is that cell throughput and hence spectral efficiency will increase.

Figure 4 shows the effect of increasing the minimum SNR threshold for admission into the cell. The stacked area plot represents the relative contributions of each MCS to the total spectral efficiency as indicated by the legend. As the minimum SNR threshold is increased, the relative contribution of lowest order MCS decreases while the 
every other MCS contribution increases. As a result the maximum spectral efficiency that can be achieved by a uniformly distributed population of users increases. This continues until only the highest order MCS remains at which point the spectral efficiency can increase no more. This represents the maximum spectral efficiency which can be achieved for the frame structure and bandwidth used.

Comparing the maximum spectral efficiency to that of the default model ( 0 increase in minimum SNR) the gain is approximately $230 \%$. In general, the cell capacity (for additional users) should exhibit a similar gain given the linear relationship between the number of users and the spectral efficiency. This provides an upper bound on the maximum number of users that can be admitted to a cell of any size given a traffic profile.

\section{b) Optimising for modified user density}

Two changes to user density are considered: decreased density and increased density. Furthermore, it is assumed that this change occurs after the cell has been deployed so that it is not possible to simply redesign the cell with an area which corresponds to $P_{\text {OSE }}$ for the revised population.

If the active user density decreases (e.g. due to day-night usage patterns) then the spectral efficiency will also decrease. With a fixed cell size it is not possible to increase the spectral efficiency by modifying transmit power in conjunction with the modified admission scheme. It is only possible to increase the spectral efficiency again by decreasing the bandwidth allocation of the cell.

Next, an increase in user density is considered. To accommodate the increased traffic demand the modified admission scheme may be used. In the absence of other constraints, the maximum increase in cell capacity that could be expected is approximately $230 \%$. However, as the minimum SNR threshold for admission increases, the serviceable area of the cell decreases if transmit power is held constant. Therefore, if the cell capacity is to increase while maintaining the same serviceable area, the transmit power must be increased by the same amount as the minimum SNR threshold is increased. Ultimately, the maximum transmit power places a limit on how much the minimum SNR value can increase. This limit corresponds to the difference between the original transmit power and the maximum allowable transmit power $(43 \mathrm{dBm})$. For this reason, the achievable gain in cell capacity depends on the transmit power prior to user density increase.

Starting with the deployment of a cell for a user density of $2 \mathrm{user} / \mathrm{km}^{2}$ and the VoIP traffic profile, $P_{\text {Opt-SE }}$ was calculated as $32 \mathrm{dBm}$. The maximum number of users admitted at this transmit power was approximately 210 . Increasing the transmit power and minimum SNR threshold in tandem and using the modified admission scheme, the maximum number of users that could be admitted was 360, a gain of approximately $71 \%$. The procedure was repeated for different initial user densities using the VoIP traffic profile and in each case maximum achievable gain was the same. The increase in the transmit power and minimum SNR threshold required to achieve this gain was also constant.

The results showed that, in addition to being limited by the maximum transmit power, the possible gain in cell capacity (for users or traffic) is also limited by the number of control resources available per LTE sub-frame. As the minimum SNR threshold increases and the cell traffic increases the number of control resources that must be allocated also increases. In LTE the number of control resources available per sub-frame is fixed and eventually, for a large number of users, all available control resources may be allocated. In this case no more traffic can be handled, despite the possible availability of unused data resources. The VoIP traffic profile is particularly testing in this regard as it permits a large number of users, each of whom transmit relatively small amounts of data. This explains why the achieved gain $(71 \%)$ is substantially lower than the theoretical maximum $(230 \%)$. It can be concluded the achievable gain in cell capacity depends not only on the initial transmit power, but also on the traffic profile or traffic mix.

Although inter-cell interference is not considered in this model it is worth noting that such interference would affect surrounding cells due to the increase in power required to increase the spectral efficiency while servicing the original cell area. Nevertheless, it is clear that the modified admission scheme can increase spectral efficiency and may be appropriate for handling certain kinds of increase in the density of active users.

\section{c) Optimising for modified cell size}

Unlike the previous section which considered changes to already deployed cell, this section considers the deployment of cells whose serviceable areas must differ from the optimum spectrally efficient area. Similar to the previous section, cells that are required to be smaller than or larger than the optimum for a given user density and traffic profile are considered.

A serviceable area smaller than the calculated optimum can be supported simply by decreasing the transmit power from $P_{\text {Opt-SE }}$. However, in addition to reducing the cell's serviceable area, this also reduces the traffic demand on the cell such that the full cell capacity is not used and the spectral efficiency is less than maximum (i.e. the valuable spectrum resource is not being used efficiently). A possible benefit of moderate inefficiency is that it provides the cell some headroom for population or traffic growth without requiring any changes to infrastructure, spectrum, or admission scheme. If the spectral efficiency is substantially less than maximum, it can be increased by reducing the bandwidth allocated to the cell.

To support a serviceable area larger than the spectrally efficient optimum, the transmit power must 
(a)

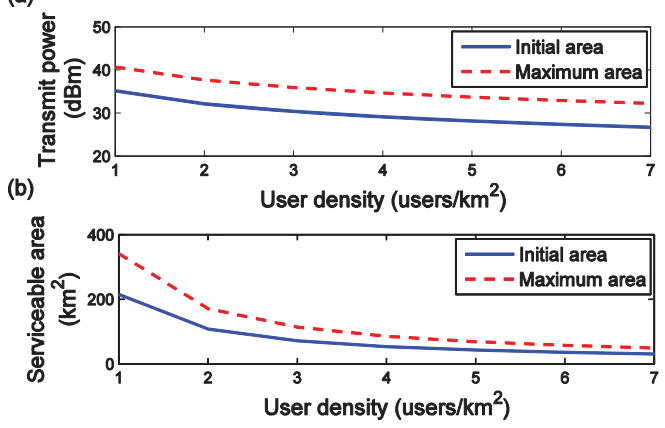

Figure 5: Increase in serviceable area from optimum versus user density

be increased by $\Delta P_{\text {area }}$ to increase cell size. The spectral efficiency must then be increased to support the increased traffic demands of the larger cell population resulting from the increased area. This increase in spectral efficiency is provided by a further increase in power, $\Delta P_{\text {SE-Increase. }}$ Therefore the total power increase required, $\Delta P_{\text {total }}$, is given by

$$
\Delta P_{\text {total }}=\Delta P_{\text {area }}+\Delta P_{S E-\text { Increase }}
$$

As before, the maximum power increase that can be supported is ultimately limited by the difference between the maximum transmit power and the initial transmit power. When the spectral efficiency of the cell is increased, the maximum cell capacity (the maximum number of users that can be admitted), $C_{\max }$, also increases (unless limited by the availability of control resources). In general, the maximum nonoptimum area, $A_{\max }$, that can be supported is given by

$$
A_{\max }=\frac{C_{\max }}{D}
$$

where $D$ is the user density. Therefore the gain in cell area is directly proportional to the gain in cell capacity.

The results in the previous section for rural user densities and the VoIP traffic profile, found that the maximum gain in cell capacity was approximately $71 \%$. It was expected that an identical area gain would be possible as long as the maximum transmit power limit was not reached. Figure 5 a shows both $P_{O p t-S E}$ and the increased power required to increase the serviceable area to its maximum for a variety of user densities. Figure 5b shows the corresponding optimum spectrally efficient area and maximum area achieved. In all cases the maximum area gain was about $58 \%$. This is somewhat lower than the predicted gain because of the discrete steps in which the transmit power and minimum SNR threshold were increased

It is worth noting that a different traffic profile would result in a different area gain. Furthermore, since two separate power increases are required to support an area larger than the spectrally efficient optimum, it may be that the area gain is more limited by the maximum transmit power than the user density gains considered in the previous section would be.

\section{CONCLUSION}

In this paper spectral efficiency was analysed in the context of a fixed bandwidth single LTE base station. It was shown that the optimum spectrally efficient transmit power for a given user density and traffic profile corresponds to the maximum transmit power which permits full user coverage. It was also shown that the optimum transmit power and cell size decreased as either user density or packet size increased. A modified admission scheme was presented to optimize the spectral efficiency of cells which experienced population/traffic growth and cells which were larger than the spectrally efficient optimum size. Although a theoretical population or area increase of approximately $230 \%$ could be supported, practical limitations in LTE interacting with the particular traffic profile (VoIP) yielded gains that were smaller than this predicted maximum.

\section{ACKNOWLEDGEMENTS}

The authors wish to thank Jean-Christophe Schiel and François Montaigne for their assistance and support. Also the authors extend thanks to the sponsors EADS and IRCSET for the PhD program.

\section{REFERENCES}

[1] Cisco, "Cisco visual networking index: Global mobile data traffic forecase update, 2011-2016," White paper, 2010.

[2] A. U. Mikko, "Global Vision for the Future Wireless World from the WWRF," Vehicular Technology Magazine, IEEE, vol. 1, pp. 4-8, 2006.

[3] A. Toskala, "Performance," in LTE for UMTS, ed: John Wiley \& Sons, Ltd, 2009, pp. 213-258.

[4] M. K. Karray, "Spectral and energy efficiencies of OFDMA wireless cellular networks," in Wireless Days (WD), 2010 IFIP, 2010, pp. 1-5.

[5] D. Bultmann, et al., "Analysis of 3GPP LTE-Advanced cell spectral efficiency," in Personal Indoor and Mobile Radio Communications (PIMRC), 2010 IEEE 21st International Symposium on, 2010, pp. 1876-1881.

[6] S.-I. Chakchai, et al., "Capacity Evaluation for IEEE 802.16e Mobile WiMAX," ed: Hindawi Publishing Corporation, 2010.

[7] T. Keenan and R. Villing, "Evaluating Wireless Cell Capacity," in RIA Colloquium on Communications and Radio Ccience into the 21st Century, 2012.

[8] R. Ratasuk, et al., "TDD design for UMTS Long-Term Evolution," in Personal, Indoor and Mobile Radio Communications, 2008. PIMRC 2008. IEEE 19th International Symposium on, 2008, pp. 1-5.

[9] M. Alshami, et al., "Evaluation of Path Loss Models at WiMAX Cell-Edge," in New Technologies, Mobility and Security (NTMS), 2011 4th IFIP International Conference on, 2011, pp. 1-5.

[10] V. Chandrasekhar, et al., "Femtocell networks: a survey," Communications Magazine, IEEE, vol. 46, pp. 59-67, 2008. 